\title{
Operational Transresistance Amplifier-Based Multiphase Sinusoidal Oscillators
}

\author{
Rajeshwari Pandey, ${ }^{1}$ Neeta Pandey, ${ }^{1}$ Mayank Bothra, ${ }^{2}$ and Sajal K. Paul ${ }^{3}$ \\ ${ }^{1}$ Department of Electronics and Communication Engineering, Delhi Technological University, Delhi, 42, India \\ ${ }^{2}$ Kritikal Solutions Pvt. Ltd., Embedded Division, Noida, UP, 201301, India \\ ${ }^{3}$ Department of Electronics Engineering, Indian School of Mines, Dhanbad, 826004, India \\ Correspondence should be addressed to Sajal K. Paul, sajalkpaul@rediffmail.com
}

Received 7 March 2011; Accepted 19 May 2011

Academic Editor: Raj Senani

Copyright ( $\odot 2011$ Rajeshwari Pandey et al. This is an open access article distributed under the Creative Commons Attribution License, which permits unrestricted use, distribution, and reproduction in any medium, provided the original work is properly cited.

\begin{abstract}
Multiphase sinusoidal oscillator circuits are presented which utilize Operational Transresistance Amplifier (OTRA) as the active element. The first circuit produces $n$ odd-phase oscillations of equal amplitudes and equally spaced in phase. The second circuit is capable of producing $n$ odd- or even- phase oscillations equally spaced in phase. An alternative approach is discussed in the third circuit, which utilizes a single-phase tunable oscillator circuit which is used to inject signals into a phase shifter circuits. An automatic gain control (AGC) circuit has been implemented for the second and third circuit. The circuits are simple to realize and have a low component count. PSPICE simulations have been given to verify the theoretical analysis. The experimental outcome corroborates the theoretical propositions and simulated results.
\end{abstract}

\section{Introduction}

Multiphase sinusoidal oscillators (MSOs) find extensive application in the field of power electronics and communications. In Communications MSO circuits are commonly used in single-sideband generators, phase modulators, and quadrature mixers. They are also utilized for control of single-phase-to-three-phase PWM converters [1] and for a decoupled dynamic control of a six-phase two-motor drive system [2]. A number of circuits are reported in the literature relating to MSOs [3-17]. The MSOs implemented in [3-7] suffer from complex circuitry. Active $R$ implementations in $[8,9]$ lack tenability, while the implementation in [10] using an OTA is tunable but has a limited output voltage swing. In $[11,12]$, MSOs based on Current Conveyor II (CC II) are presented. Their drawback is that they require a JFET and three additional current conveyors for each phase in order to achieve electronic tunability [13]. The structures proposed in $[13,14]$ utilize translinear CC, and those presented in $[15,16]$ are based on Current Differencing Transconductance Amplifier (CDTA). Though these circuits can operate at high frequencies and provide electronic tunability, they provide current outputs which need to be converted back to voltage for circuits requiring voltage inputs, which would considerably increase the component count. Moreover, the implementations utilizing parasitic resistors $[13,14]$ are not very accurate in producing the desired phase shift. The structure proposed in [17] using Current Feedback Operational Amplifier (CFOA) has a good output voltage swing and capable of producing high frequencies but requires an accessible compensation terminal of a CFOA. A major difficulty that is observed with a self-oscillating MSO is the stabilization of the signal amplitude. There are a number of techniques available for controlling amplitude of oscillations [18] for voltage mode op-amps. However, these techniques cannot be applied readily to current mode op-amps.

It is well known that the key performance features of current mode technique are inherent wide bandwidth which is virtually independent of closed loop gain, greater linearity, and large dynamic range [19]. Recently Operational Transresistance Amplifier (OTRA) has emerged as an effective alternate analog building block which is a high gain current input, voltage output amplifier [20]. OTRA, being a current processing analog building block, inherits all the advantages of current mode technique and therefore is ideally suited for 


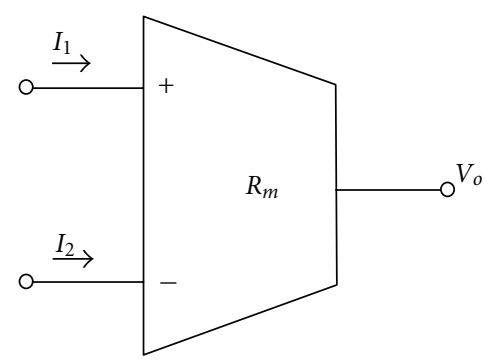

FIgURE 1: OTRA circuit symbol.

high-frequency applications [21]. It is also free from parasitic input capacitances and resistances as its input terminals are virtually grounded, and, hence, nonideality problem is less in circuits implemented with OTRA.

This paper presents OTRA-based three MSO circuits, which are described in Section 2. The first circuit utilizes $n$ OTRAs to produce $n$ odd-phase oscillations of equal amplitudes and equally spaced in phase. The second circuit utilizes $(n+1)$ OTRAs to produce $n$ odd- or even- phase oscillations equally spaced in phase. The third circuit utilizes a single-resistance-controlled (SRCO) sinusoidal oscillator circuit employing a single OTRA [22], whose output is subsequently used to drive a phase shifter network. The phase shifter circuit uses $n$ OTRA-based phase shifter blocks to give a total of $(n+1)$ oscillations. The circuit is tunable and has a low component count. An Automatic Gain Control circuitry (AGC) has also been implemented for the second and third circuits, which helps in the stabilization of the signal amplitude. Section 3 deals with nonideal analysis of the OTRA, while Section 4 gives the simulation and experimental results of the proposed MSO circuits. Conclusion is discussed in Section 5.

\section{Circuit Description}

The circuit symbol of OTRA is shown in Figure 1, and port relationship can be given by

$$
\left[\begin{array}{c}
V_{1} \\
V_{2} \\
V_{O}
\end{array}\right]=\left[\begin{array}{ccc}
0 & 0 & 0 \\
0 & 0 & 0 \\
R_{m} & -R_{m} & 0
\end{array}\right]\left[\begin{array}{c}
I_{1} \\
I_{2} \\
I_{O}
\end{array}\right] .
$$

Ideally the transresistance gain $R_{m}$ approaches infinity [19, 20], and, hence, when used with negative feedback, currents at the two input terminals are forced to be equal. Both of the input terminals are virtually grounded. The output voltage is the difference of two input currents multiplied by transresistance gain $\left(R_{m}\right)$. Inputs of OTRA are current signals while the output is a voltage signal, and, consequently, as both the input and output terminals have low impedances, OTRA is suitable for cascading.

2.1. Circuit I. The first circuit is shown in Figure 2 which produces $n$ odd-phase oscillations. It is based on the scheme discussed in [23] and has been adapted for implementation with OTRA. The OTRAs have been connected in the inverting mode such that the gain $G(s)$ of each block can be expressed as

$$
G(\mathrm{~s})=\left(-\frac{K}{1+s C R}\right),
$$

where $R_{2}=R_{4}=\cdots=R_{2 n}=R, R_{1}=R_{3}=\cdots=R_{2 n-1}=$ $R_{x}, K=\left(R / R_{X}\right)$, and $C_{1}=C_{2}=\cdots=C_{n}=C$.

From Figure 2, the open loop gain $L(s)$ can be expressed as:

$$
L(s)=\left(-\frac{K}{1+s C R}\right)^{n} .
$$

For oscillations to occur, the Barkhausen criterion [18] must be satisfied, hence

$$
\left(-\frac{K}{1+s C R}\right)^{n}=1 .
$$

The above equation yields

$$
(1+s C R)^{n}+(-1)^{n+1} K^{n}=0 .
$$

Equation (5) will converge only for odd values of $n$ such that $n \geq 3$. Thus, the circuit will give rise to equally spaced oscillations having a phase difference of $(360 / n)^{\circ}$.

Consider the case for $n=3$, then (5) reduces to

$$
\left(1+j \omega_{0} C R\right)^{3}+K^{3}=0 .
$$

Equating real and imaginary parts of (5) gives the frequency of oscillation (FO) and condition of oscillation (CO) as

$$
\begin{aligned}
& \text { FO : } f_{0}=\frac{\sqrt{ } 3}{(2 \pi R C)}, \\
& \text { CO : } \quad K=2 .
\end{aligned}
$$

Similarly, for $n=5$, (5) would reduce to

$$
\left(1+j \omega_{0} C R\right)^{5}+K^{5}=0 .
$$

Hence, $\mathrm{FO}$ and $\mathrm{CO}$ can be obtained as

$$
\begin{aligned}
& \text { FO : } f_{0}=\frac{0.727}{(2 \pi R C)}, \\
& \text { CO : } \quad K=1.236 .
\end{aligned}
$$

Thus, FO and CO for any value of $n$ can be obtained. It is evident that $\mathrm{CO}$ and $\mathrm{FO}$ cannot be independently controlled for circuit $I$. However, the circuit is simple to realize and has a low component count. This circuit produces $n$ odd-phase oscillations of equal amplitudes with a phase difference of $(360 / n)^{\circ}$.

2.2. Circuit II. The second circuit is shown in Figure 3. This implementation is capable of producing $n$ odd- or evenphase oscillations. It consists of $n$ cascaded OTRA blocks, each block implementing a noninverting first-order low-pass function. An inverting voltage amplifier with a simple AGC circuit is connected in the feedback loop of the oscillator. The 


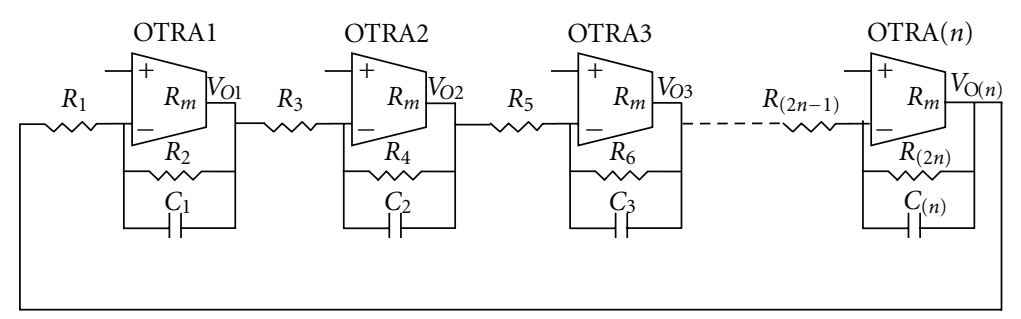

FIGURE 2: Generalized scheme for producing $n$ odd-phase oscillation.

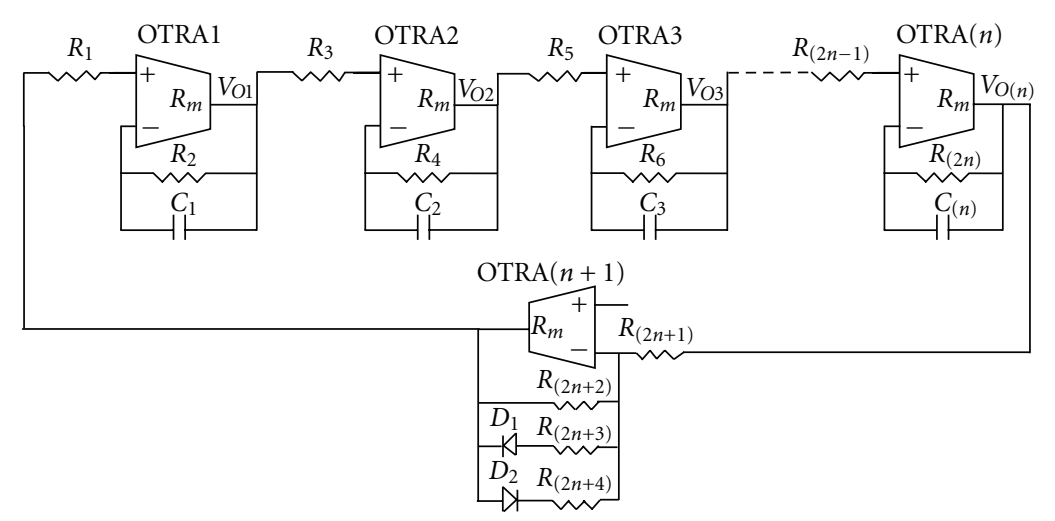

FIGURE 3: Generalized scheme for producing $n$ odd- or even- phase oscillations with AGC.

AGC circuit consists of resistors $R_{2 n+3}, R_{2 n+4}$ and two diodes $D_{1}$ and $D_{2}$.

Taking $R_{2}=R_{4}=\cdots=R_{2 n}=R, R_{1}=R_{3}=R_{2 n-1}=$ $R_{x}, \quad K=\left(R / R_{X}\right)$, and $C_{1}=C_{2}=\cdots=C_{n}=C$, gain of each block and loop gain can be computed as

$$
\begin{aligned}
G(s) & =\left(\frac{K}{1+s C R}\right), \\
L(s) & =\left(-\frac{K}{1+s C R}\right)^{n} K_{X},
\end{aligned}
$$

where

$$
K_{X}=\frac{R_{2 n+2}}{R_{2 n+1}} .
$$

$K_{X}$ is effectively maintained at a value 1 with the help of the AGC circuitry. After applying the Barkhausen criterion, the characteristic equation is obtained as

$$
(1+s C R)^{n}+K^{n}=0 .
$$

The equation converges for all value of $n \geq 3$, odd or even. As an example for $n=4,(12)$ reduces to

$$
\left(1+j \omega_{0} C R\right)^{4}+K^{4}=0 .
$$

which gives $\mathrm{FO}$ and $\mathrm{CO}$ as

$$
\begin{aligned}
& \text { FO : } f_{0}=\frac{1}{(2 \pi R C)}, \\
& \text { CO : } \quad K=1.414 .
\end{aligned}
$$

For $n=3$, the characteristic equation, $\mathrm{FO}$ and $\mathrm{CO}$ would be the same as (6), (7), and (8) respectively.

Initially $K_{X}$ is kept at a value slightly higher than 1 so that the oscillations can begin; once the amplitude crosses a certain threshold, the diodes get switched on and bring down the value of resistance $R_{2 n+2}$, thus bringing down the effective value of $K_{X}$. This is possible because the input terminals of the OTRA are virtually grounded. Thus, a dynamic equilibrium maintains the value of $K_{X}$ at 1 . This circuit is not tunable as $\mathrm{CO}$ and FO are not independent. However, the circuit is versatile and can achieve both evenand odd- phase oscillations. The oscillations achieved are equally spaced in phase having a phase difference of $(180 / n)^{\circ}$.

2.3. Circuit III. The third circuit is shown in Figure 4. It is based on an SRCO oscillator [22] to which an AGC circuit has been added which works on the same principle as in circuit II.

The open loop gain of this circuit [22] is obtained as

$$
L(s)=\left(\frac{s C_{2} R_{2} R_{3}\left(1-s C_{1} R_{1}\right)}{R_{1}+R_{3}+s R_{1} R_{3}\left(C_{1}+C_{2}\right)}\right) .
$$

Accordingly, the FO and CO for SRCO oscillator are obtained as

$$
\begin{aligned}
& \text { FO : } f_{0}=\frac{1}{2 \pi} \sqrt{\frac{R_{1}+R_{3}}{C_{1} C_{2} R_{1} R_{2} R_{3}}}, \\
& \text { CO : } \quad \frac{R_{2}}{R_{1}}=\frac{C_{1}}{C_{2}}+1 .
\end{aligned}
$$

It can be observed that by controlling $R_{3}$ the frequency can be controlled without affecting the CO, which makes the circuit 


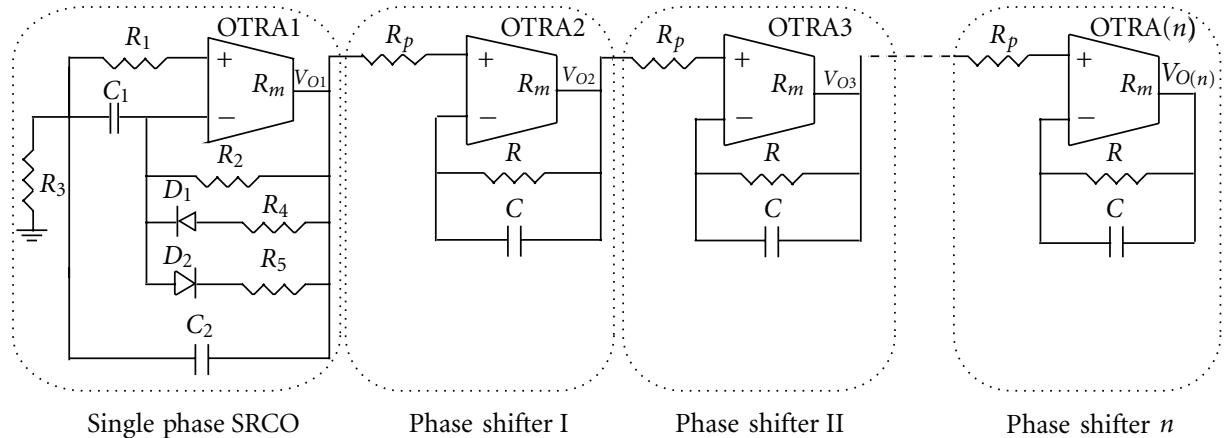

Figure 4: SRCO-based MSO.

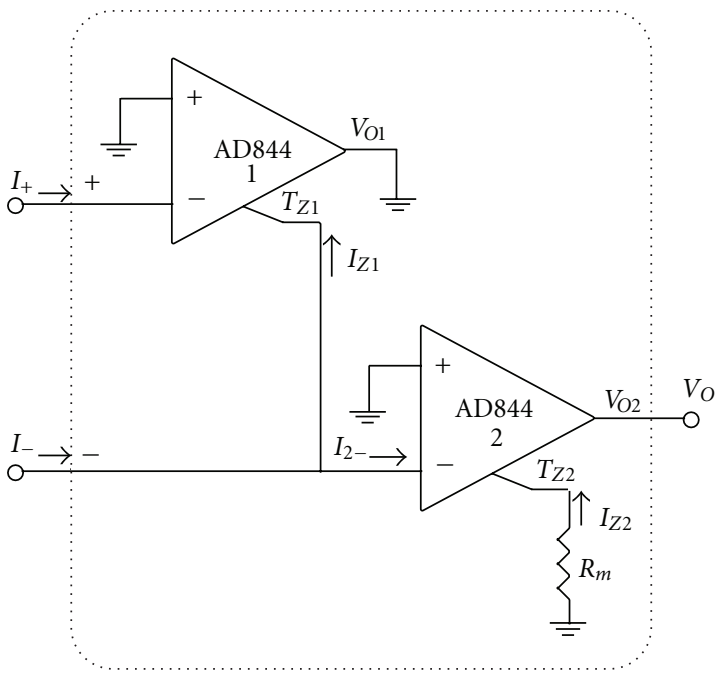

Figure 5: OTRA based on AD844.

tunable. AGC has been achieved by adjusting $R_{2}$, which is used to control the loop gain, as seen from (16). At the output of the SRCO with AGC, $n$ subsequent OTRA-based phase shifter blocks can be connected to produce $n+1$ oscillation. The phase shift produced by each phase shifter block can be given as

$$
\theta=\tan ^{-1}\left(\omega_{0} R C\right) .
$$

To add to the flexibility, if the OTRA in the phase shifter block is connected in inverting mode, the phase shift produced will be

$$
\theta=180^{\circ}-\tan ^{-1}\left(\omega_{0} R C\right) .
$$

Hence, the phase shifter can be adjusted to obtain a phase shift of either $0^{\circ}-90^{\circ}$ or $90^{\circ}-180^{\circ}$ depending on its configuration. This circuit provides the flexibility of achieving the desired phase shift without connecting too many active elements.

\section{Nonideality Analysis}

The nonidealities associated with OTRA-based circuits may be divided into two groups. The first group concerns with

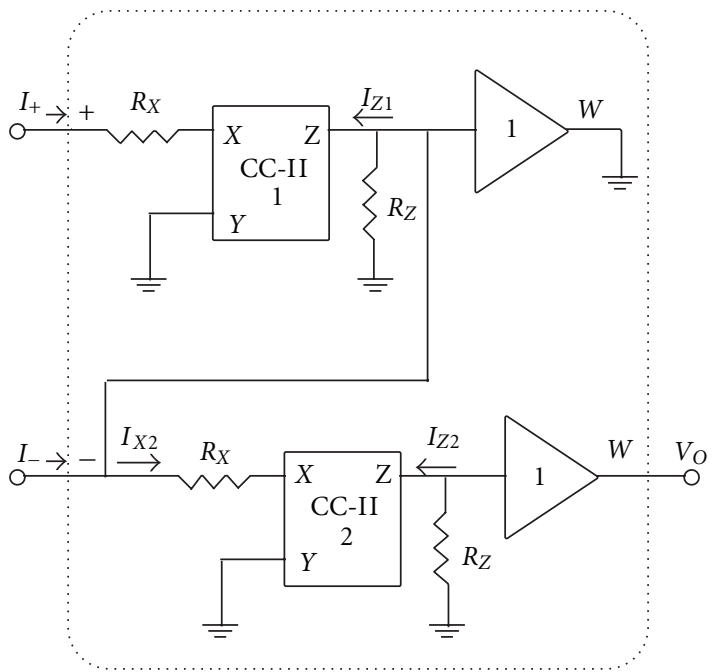

FIGURE 6: Equivalent circuit of OTRA constructed with AD844.

finite transresistance gain, whereas the second one results from practical implementation based on commercially available IC AD 844.

3.1. Nonideality due to Finite Transresistance Gain. Here the effect of finite transresistance gain on MSO is considered, and for high-frequency applications self-compensation is employed [20]. Ideally the transresistance gain $R_{m}$ is assumed to approach infinity. However, practically $R_{m}$ is a frequencydependent finite value. Considering a single pole model for the transresistance gain, $R_{m}$ can be expressed as

$$
R_{m}(s)=\left(\frac{R_{0}}{1+s / \omega_{0}}\right)
$$

For high-frequency applications, the transresistance gain $R_{m}(s)$ reduces to

$$
R_{m}(s) \approx\left(\frac{1}{s C_{p}}\right), \quad \text { where } C_{p}=\frac{1}{R_{0} \omega_{0}} .
$$

Taking this effect into account (2) modifies to

$$
G(s)=\left(\frac{-K}{1+s\left(C+C_{p}\right) R}\right) .
$$




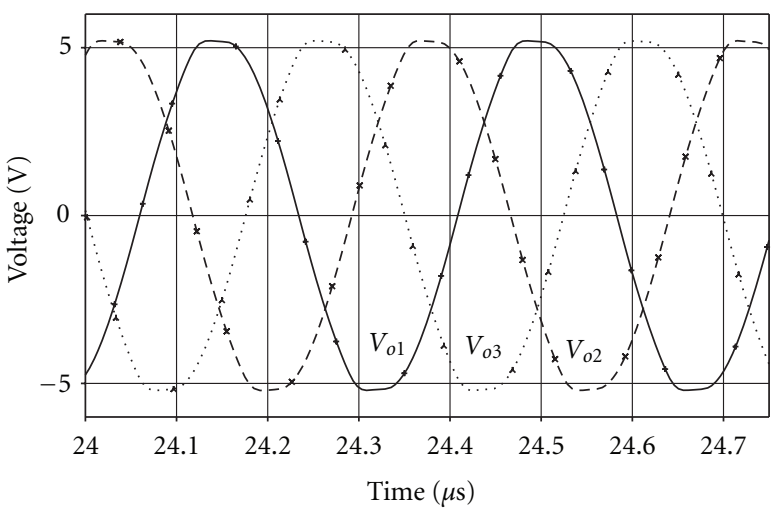

(a) Output waveform for $n=3, f=2.838 \mathrm{MHz}$

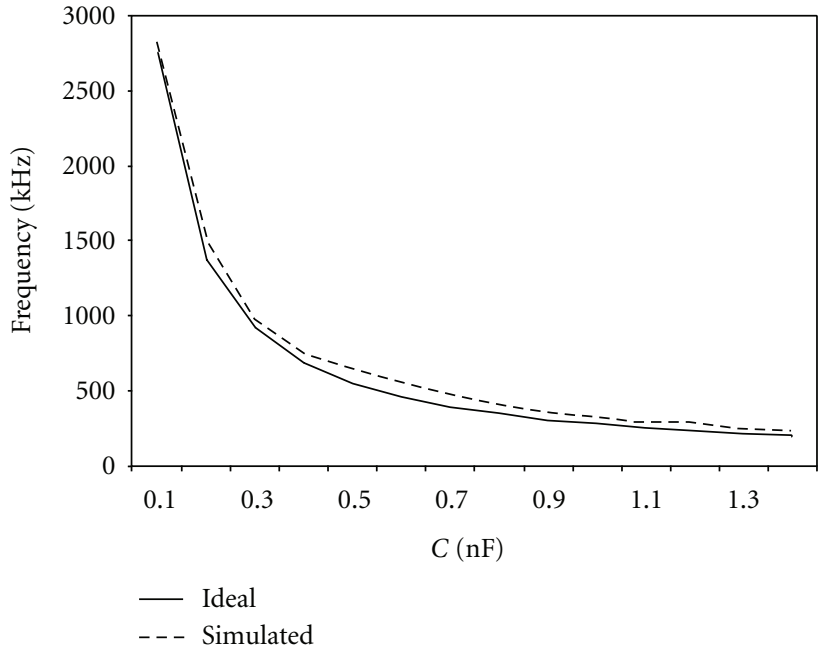

(b) Frequency error curve

FIGURE 7: Simulation result for circuit I.

The effect of $C_{p}$ can be eliminated by preadjusting the value of designed capacitor and thus achieving self-compensation.

\subsection{Nonideality Attributed to Realization of OTRA Using} Commercially Available AD844 [24]. The OTRA can be realized using AD844 CFOA IC as shown in Figure 5 [24]. Figure 6 [24] shows the equivalent circuit for nonideal analysis of the circuit presented in Figure 5. The CFOAs have been replaced with current conveyors having finite input resistances $\left(R_{X}\right)$ and finite resistance at its $Z$ terminal $\left(R_{Z}\right)$. Ideally the input resistance at the $X$ terminal is zero and is infinite at the $Z$ terminal. For the AD844 CFOA the input resistance $R_{X}$ is around $50 \Omega$ and $R_{Z}$ is around $3 \mathrm{M} \Omega$ [25]. From Figure 6 various currents can be calculated as follows:

$$
\begin{aligned}
I_{Z 1} & =I_{+}, \\
I_{X 2} & =I_{D}-I_{-}, \\
I_{D} & =I_{Z 1}\left(\frac{R_{Z}}{R_{X}+R_{Z}}\right), \\
I_{Z 2} & =I_{X 2} .
\end{aligned}
$$

$R_{Z}$ is the transimpedance of the OTRA. Ideally $I_{D}$ should be equal to $I_{Z 1}$, which can be approximated only if $R_{Z}$ is much greater than $R_{X}$, which is true for AD844. Also the approximation that the input terminals are virtually grounded will be true only if the external resistance at the input terminal of the OTRA is much larger than $R_{X}$.

If these two conditions are satisfied, the OTRA constructed with AD844 closely approximates an ideal OTRA. From (23) the output voltage $V_{O}$, taking into account the above-mentioned approximations, can be calculated as

$$
V_{O}=\left(I_{+}-I_{-}\right) R_{Z}
$$

\section{Simulation and Experimental Results}

The proposed circuits have been simulated using PSPICE to validate the theoretical predictions. The OTRA is realized using IC AD 844 as shown in Figure 5. Figure 7(a) shows the simulation results of circuit I having $n=3$ and component values $R_{1}=R_{3}=R_{5}=0.5 \mathrm{k} \Omega, R_{2}=$ $R_{4}=R_{6}=1 \mathrm{k} \Omega$, and $C_{1}=C_{2}=C_{3}=100 \mathrm{pF}$. The frequency of oscillations achieved was $2.838 \mathrm{MHz}$ against the calculated value of $2.757 \mathrm{MHz}$ having frequency error of $2.93 \%$. Figure $7(\mathrm{~b})$ shows the simulated and theoretical frequency of oscillation as a function of capacitance $(C)$. It shows that the simulated values deviate slightly from the ideal values at lower frequency range.

Figure 8 (a) shows the simulation results of circuit II having $n=4$ and component values $R_{1}=R_{3}=R_{5}=$ $R_{7}=0.707 \mathrm{k} \Omega, R_{2}=R_{4}=R_{6}=R_{8}=R_{9}=R_{10}=$ $1 \mathrm{k} \Omega$, and $C_{1}=C_{2}=C_{3}=C_{4}=100 \mathrm{pF}$. The simulated value achieved was $1.595 \mathrm{MHz}$ against the theoretical value of $1.591 \mathrm{MHz}$ with a frequency error of $0.25 \%$. Figure $8(\mathrm{~b})$ shows the simulated and theoretical frequency of oscillation as a function of capacitance $(C)$.

Figure 9(a) shows the simulation results of circuit III having two phase shifter blocks along with the single phase oscillator (SRCO). The design is obtained for an FO of $2.220 \mathrm{MHz}$ with a phase shift of $45^{\circ}$. The component values chosen are $R_{1}=R_{3}=R_{p}=1 \mathrm{k} \Omega, R_{2}=2 \mathrm{k} \Omega, C_{1}=C_{2}=$ $C=50 \mathrm{pF}$, and $R=1.434 \mathrm{k} \Omega$. The frequency observed was $2.220 \mathrm{MHz}$ against the calculated value of $2.361 \mathrm{MHz}$. The simulated and theoretical frequency of oscillation as a function of capacitance (C) is shown in Figure 9(b). It may be observed from Figures 7(b), 8(b), and 9(b) that the deviation between the simulated and theoretical FO is minimum in the case of circuit III.

The functionality of the proposed MSO circuits is verified through hardware also. The commercial IC AD844AN is used to implement an OTRA. Supply voltages used are $\pm 5 \mathrm{~V}$. 


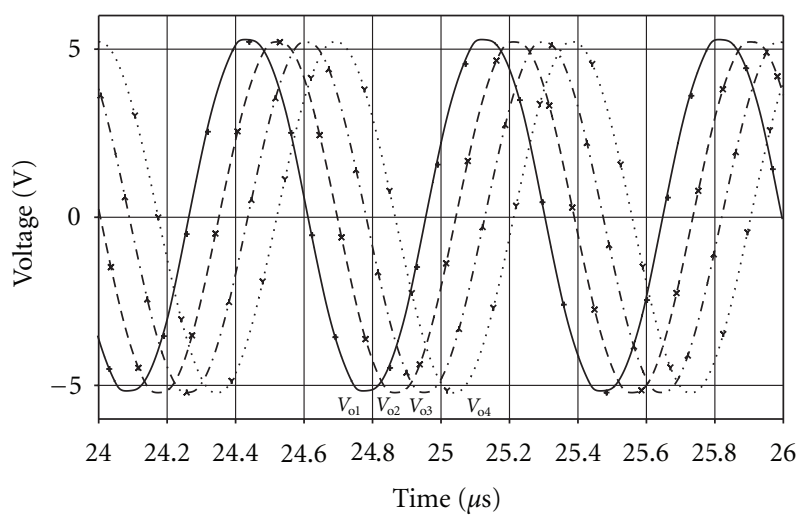

(a) Output waveforms for $n=4, f=1.546 \mathrm{MHz}$

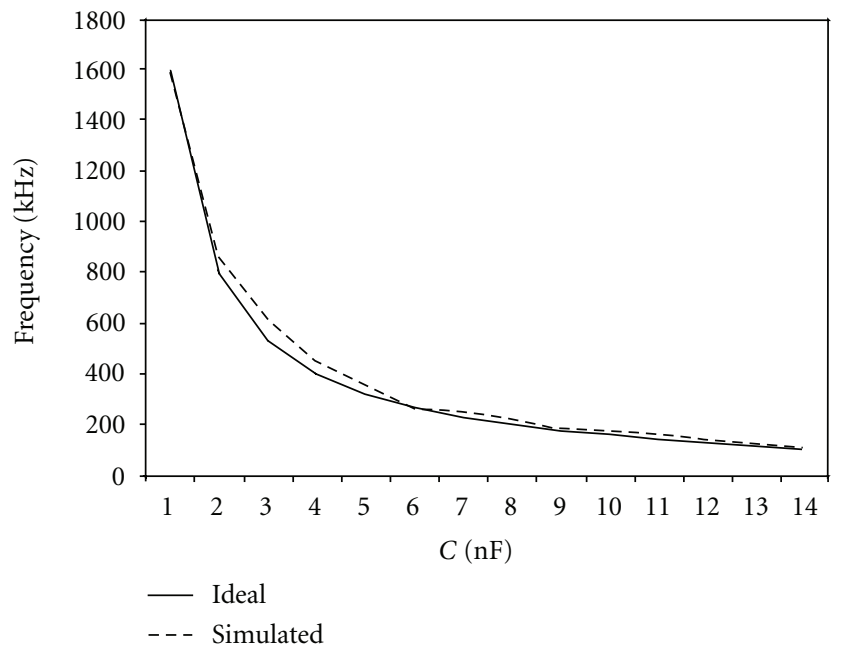

(b) Frequency error curve

FIGURE 8: Simulation results for circuit II.

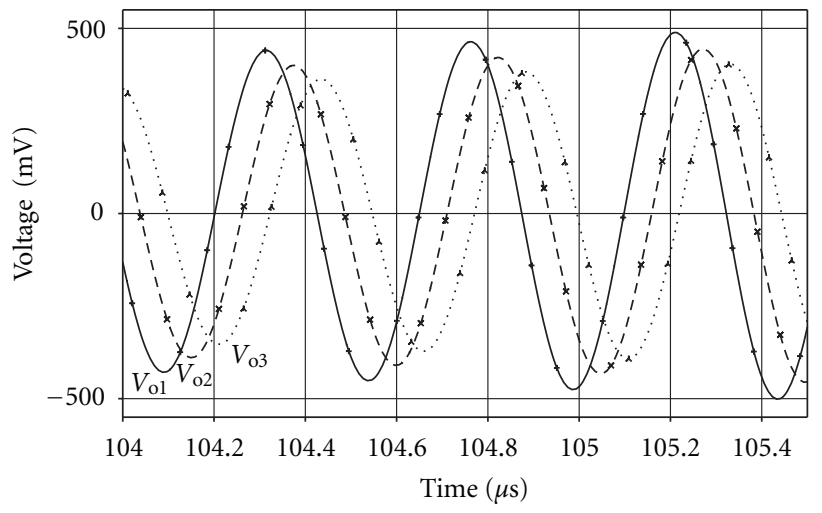

(a) Output waveforms having 2 phase shifter networks of $45^{\circ}$ each, $f=$ $1.401 \mathrm{MHz}$

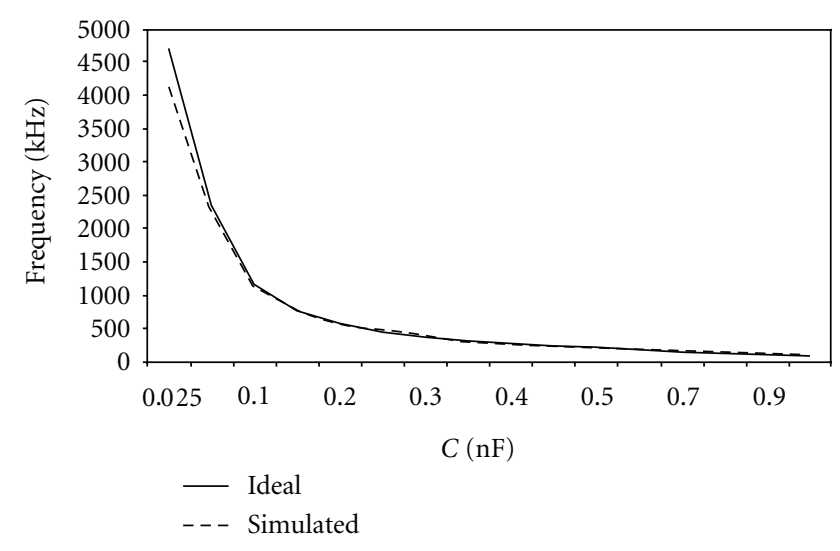

(b) Frequency error curve

FIGURE 9: Simulation result for circuit III.

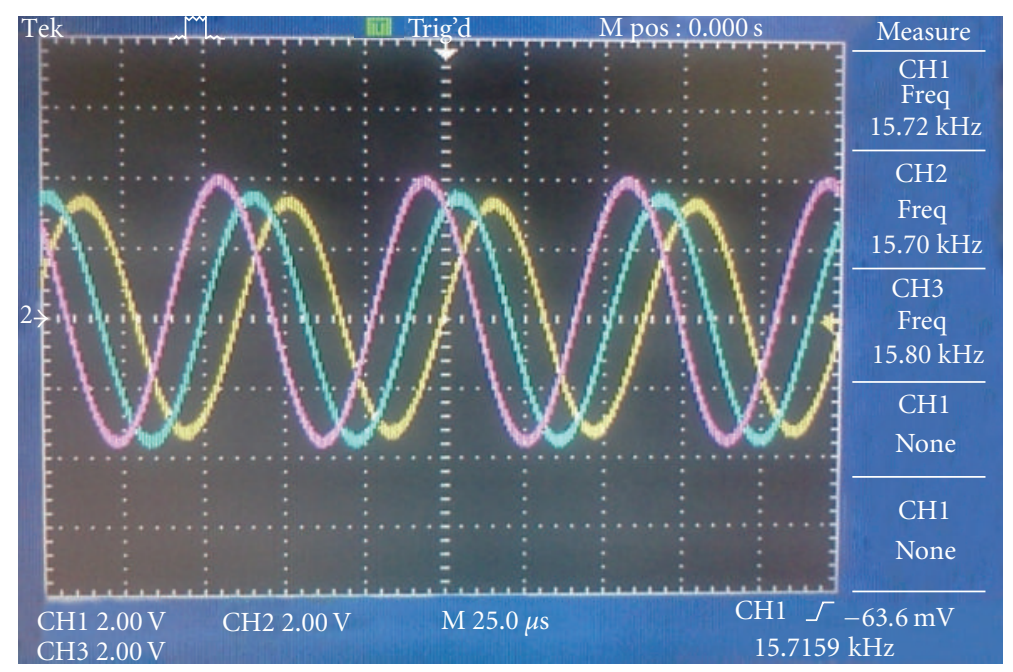

Figure 10: Experimental result for circuit II for $n=3$. 


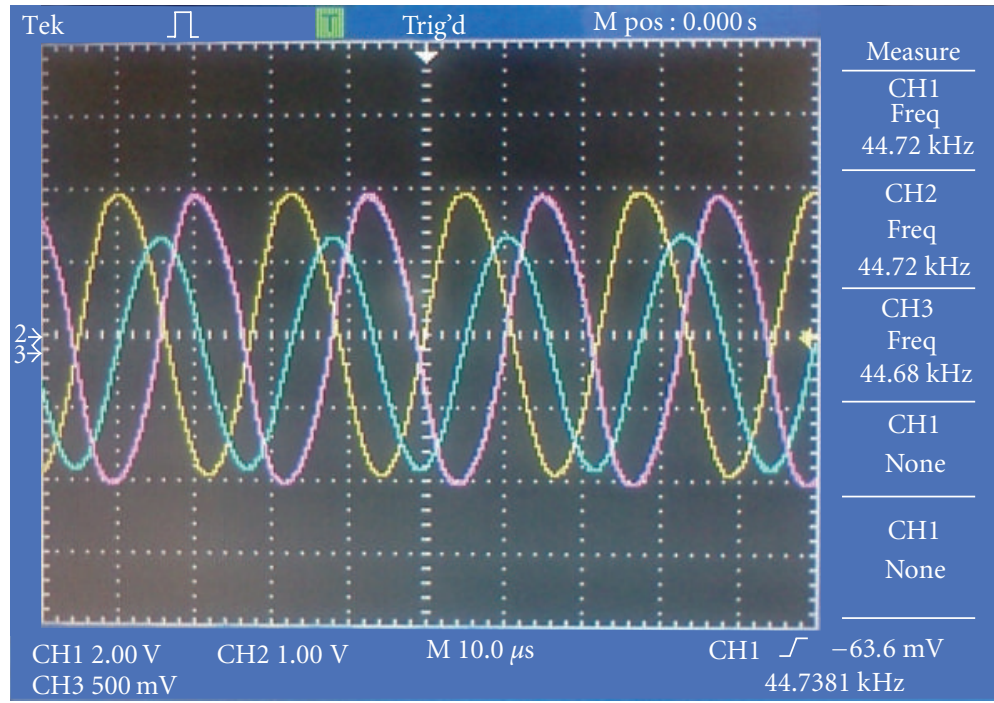

Figure 11: Experimental result for circuit III with two phase shifter blocks.

Figure 10 shows the experimental results for circuit II, having $n=3$, for component values $R_{1}=R_{3}=R_{5}=2.7 \mathrm{k} \Omega$, $R_{2}=R_{4}=R_{6}=5.4 \mathrm{k} \Omega, R_{8}=R_{9}=R_{10}=1 \mathrm{k} \Omega$, and $C_{1}=C_{2}=C_{3}=3.3 \mathrm{nF}$. Observed FO is around $15.7 \mathrm{kHz}$ and is in close agreement with calculated FO of $15.469 \mathrm{kHz}$. The little variation in experimental values of FO, as seen in Figure 10, from phase to phase may be due to tolerance of the component values.

The output of circuit III consisting of SRCO along with two phase shifter blocks is depicted in Figure 11 for component values $R_{1}=R_{p}=2.7 \mathrm{k} \Omega, R_{2}=5.4 \mathrm{k} \Omega$, $R_{3}=250 \Omega, R=10 \mathrm{k} \Omega$, and $C=C_{1}=C_{2}=3.3 \mathrm{nF}$. These component values result in theoretical FO as $43.4 \mathrm{kHz}$, and the observed frequency is around $44.72 \mathrm{kHz}$. The little variation in the experimental values of FO from phase to phase may be due to tolerance of the component values.

\section{Conclusion}

Three MSO circuits based on OTRAs have been presented. The circuits are versatile and have a wide range of frequency of oscillation. They provide advantages of current mode design techniques and at the same time provide voltage outputs. Hence, they are capable of replacing voltage mode op-amps-based MSO since they can provide higher frequencies of oscillation and are free from the drawbacks of conventional voltage mode op-amps. These circuits are very accurate in providing the desired phase shift without utilizing too many components. They provide high output voltage swing at frequencies in the $\mathrm{MHz}$ range. The workability of these circuits has been demonstrated through SPICE simulations and experimental results.

\section{References}

[1] D. C. Lee and Y. S. Kim, "Control of single-phase-to-threephase AC/DC/AC PWM converters for induction motor drives," IEEE Transactions on Industrial Electronics, vol. 54, no. 2, pp. 797-804, 2007.

[2] M. Jones, S. N. Vukosavic, E. Levi, and A. Iqbal, "A six-phase series-connected two-motor drive with decoupled dynamic control," IEEE Transactions on Industry Applications, vol. 41, no. 4, pp. 1056-1066, 2005.

[3] A. Rahman and S. E. Haque, "A simple three-phase variablefrequency oscillator," International Journal of Electronics, vol. 53, no. 1, pp. 83-89, 1982.

[4] V. P. Ramamurthi and B. Ramaswami, "A novel three-phase reference sine wave generator for PWM invertors," IEEE Transactions on Industrial Electronics, vol. IE-29, no. 3, pp. 235-240, 1982.

[5] R. Rabinovici, B. Z. Kaplan, and D. Yardeni, "Fundamental topologies of three-phase LC resonators and their applications for oscillators," Proceedings Institution of Electrictronic Engineering, vol. 140, part G, no. 3, pp. 148-154, 1993.

[6] W. B. Mikhael and S. Tu, "Continuous and switched-capacitor multiphase oscillators," IEEE Transactions on Circuits and Systems, vol. CAS-31, no. 3, pp. 280-293, 1984.

[7] B. Z. Kaplan and S. T. Bachar, "A versatile voltage controlled three phase oscillator," IEEE Transactions on Industrial Electronics and Control Instrumentation, vol. IECI- 26, no. 3, pp. 192-195, 1979.

[8] M. T. Abuelma'atti and W. A. Almansoury, "Active-R multiphase oscillators," Proceedings Institution of Electrictronic Engineering, vol. 134, part G, no. 6, pp. 292-293, 1987.

[9] D. Stiurca, "On the multiphase symmetrical active-R oscillators," IEEE Transactions on Circuits and Systems II, vol. 41, no. 2, pp. 156-158, 1994.

[10] I. A. Khan, M. T. Ahmed, and N. Minhaj, "Tunable OTAbased multiphase sinusoidal oscillators," International Journal of Electronics, vol. 72, no. 3, pp. 443-450, 1992.

[11] D. S. Wu, S. I. Liu, Y. S. Hwang, and Y. P. Wu, "Multiphase sinusoidal oscillator using second-generation current conveyors," International Journal of Electronics, vol. 78, no. 4, pp. 645651, 1995.

[12] C. L. Hou and B. Shen, "Second-generation current conveyorbased multiphase sinusoidal oscillators," International Journal of Electronics, vol. 78, no. 2, pp. 317-325, 1995. 
[13] M. T. Abuelma'Atti and M. A. Al-Qahtani, "A new currentcontrolled multiphase sinusoidal oscillator using translinear current conveyors," IEEE Transactions on Circuits and Systems II, vol. 45, no. 7, pp. 881-885, 1998.

[14] C. Loescharataramdee, W. Kiranon, W. Sangpisit, and W. Yadum, "Multiphase sinusoidal oscillators using translinear current conveyors and only grounded passive components," in Proceedings of the 33rd Southeastern Symposium on System Theory, pp. 59-63, 2001.

[15] W. Tangsrirat and W. Tanjaroen, "Current-mode multiphase sinusoidal oscillator using current differencing transconductance amplifiers," Circuits, Systems, and Signal Processing, vol. 27, no. 1, pp. 81-93, 2008.

[16] W. Tangsrirat, W. Tanjaroen, and T. Pukkalanun, "Currentmode multiphase sinusoidal oscillator using CDTA-based allpass sections," AEU-International Journal of Electronics and Communications, vol. 63, no. 7, pp. 616-622, 2009.

[17] D. S. Wu, S. I. Liu, Y. S. Hwang, and Y. P. Wu, "Multiphase sinusoidal oscillator using the CFOA pole," Proceedings Institution of Electrictronic Engineering: Circuits, Devices and Systems, vol. 142, no. 1, pp. 37-40, 1995.

[18] A. S. Sedra and K. C. Smith, Microelectronic Circuits, Oxford University, New York, NY, USA, 2004.

[19] C. Toumazou, F. J. Lidgey, and D. G. Haigh, Analogue IC Design: The Current Mode Approach, chapter 1, Peregrinus, London, UK, 1990.

[20] J. Chen, H. Tsao, and C. Chen, "Operational transresistance amplifier using CMOS technology," Electronics Letters, vol. 28, no. 22, pp. 2087-2088, 1992.

[21] K. N. Salama and A. M. Soliman, "CMOS operational transresitance amplifier for analog signal processing applications," Microelectronics Journal, vol. 30, pp. 235-245, 1999.

[22] U. Cam, "A novel single-resistance-controlled sinusoidal oscillator employing single operational transresistance amplifier," Analog Integrated Circuits and Signal Processing, vol. 32, no. 2, pp. 183-186, 2002.

[23] S. Gift, "Multiphase sinusoidal oscillator using invertingmode operational amplifiers," IEEE Transactions on Instrumentation and Measurement, vol. 47, no. 4, pp. 986-991, 1998.

[24] Y. K. Lo, H. C. Chien, and H. G. Chiu, "Switch controllable OTRA based bistable multivibrator," IET Circuits, Devices and Systems, vol. 2, no. 4, pp. 373-382, 2008.

[25] AD844 Datasheet, Analog Devices Inc. 

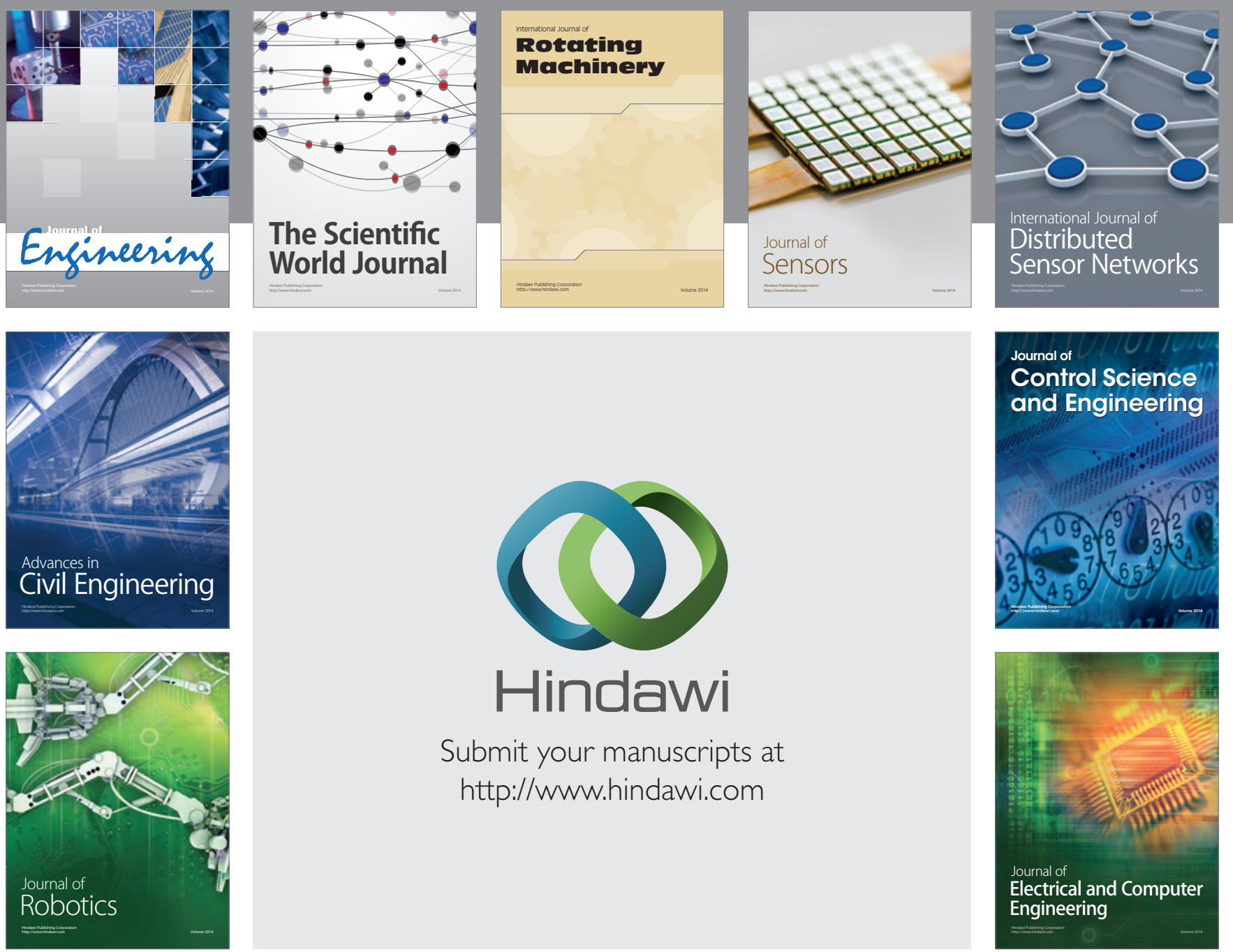

Submit your manuscripts at

http://www.hindawi.com
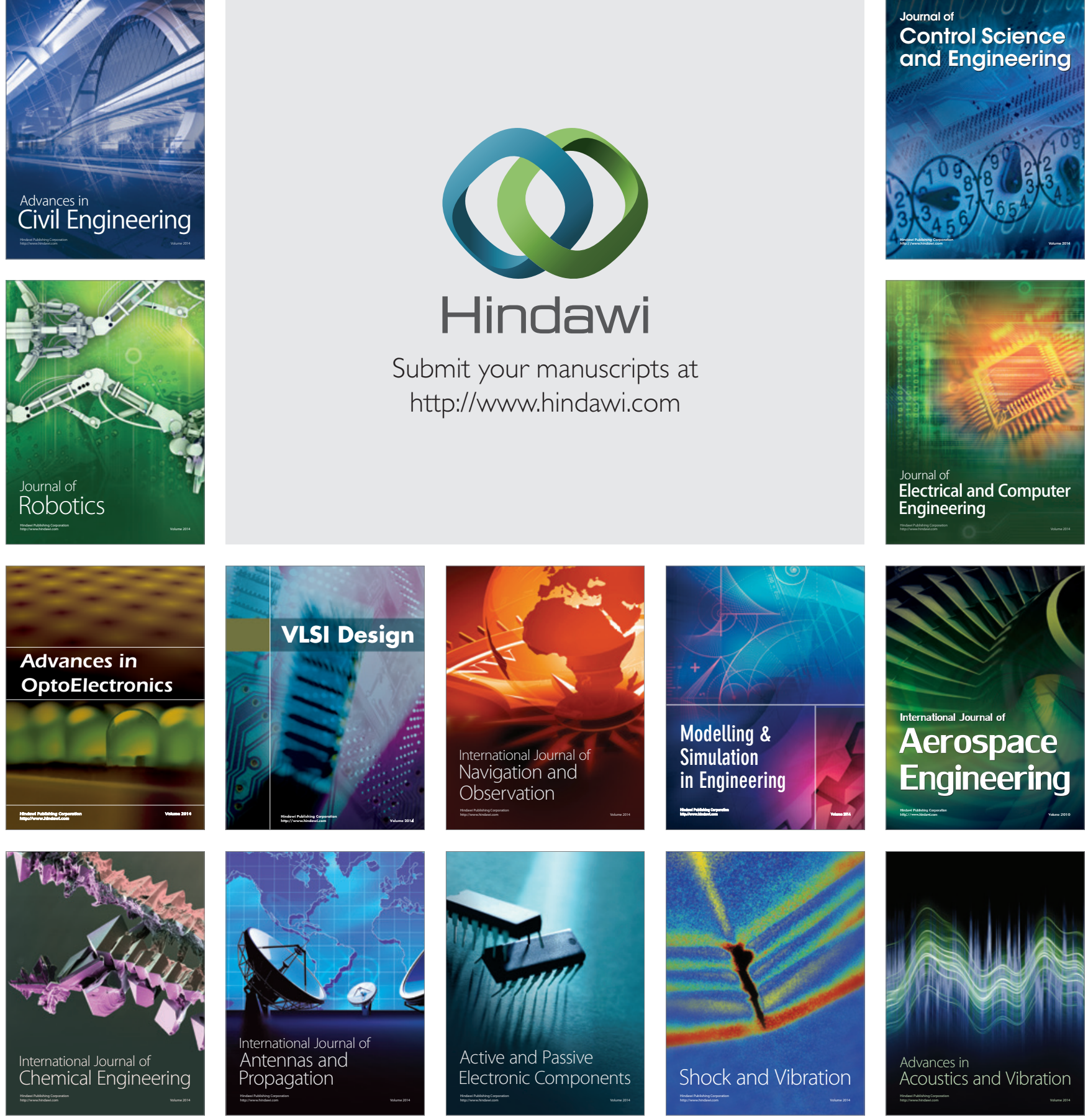